UTPT-98-05

\title{
Scale of Leptogenesis
}

\author{
Jacqueline Faridani ${ }^{(a)}$, \& Smaragda Lola ${ }^{(b)}$, \& \\ Patrick J. O'Donnell ${ }^{(c)}$ ? and Utpal Sarkar ${ }^{(d)}$ 田
}

(a) Department of Statistics and Actuarial Science, University of Waterloo, Waterloo, Ont. N2L 3G1, Canada.

(b) Theory Division, CERN, CH-1211 Switzerland, Geneva

(c) Physics Department, University of Toronto, Toronto, Ontario M5S 1A7, Canada

(d)Theory Group, Physical Research Laboratory, Ahmedabad, 380 009, India

\begin{abstract}
We study the scale at which one can generate the lepton asymmetry of the universe which could then get converted to a baryon asymmetry during the electroweak phase transition. We consider the possibility that the Yukawa couplings are small but sufficiently large to generate enough lepton asymmetry. This forbids the possibility of the $(B-L)$ breaking scale being the electroweak scale.
\end{abstract}

\footnotetext{
1jafarida@mercator.math.uwaterloo.ca

${ }^{2}$ magda@mail.cern.ch

${ }^{3}$ pat@medb.physics.utoronto.ca

${ }^{4}$ utpal@prl.ernet.in
} 


\section{Introduction}

In most grand unified theories (GUTs) the baryon asymmetry of the universe is generated during the GUT phase transition [1, 2, 3]. In these models the generated asymmetry also implies an equal amount of lepton asymmetry and hence there is no net $(B-L)$ asymmetry. On the other hand if the electroweak phase transition is a second order phase transition, then any primordial $(B+L)$ asymmetry generated during the GUT phase transition will be washed out [4] .

This situation can be saved in models where $(B-L)$ is broken at some intermediate scales. In this case a $(B-L)$ asymmetry can be generated through higgs decay or heavy Majorana neutrino decay if there is appropriate $C P$ violation [5]-[11]. The out-of-equilibrium condition then imposes a lower bound on this symmetry breaking scale to be around $10^{7} \mathrm{GeV}$. This bound is dependent on the fact that the Yukawa couplings are larger than $10^{-5}$. Although esthetically this number sounds reasonable, nothing tells us definitely that the Yukawa couplings cannot be smaller than this. For example, if the Yukawa couplings relating the left handed leptons to the first generation right handed heavy neutrinos are similar to the Yukawa couplings for the electron, then the out-of-equilibrium condition can be satisfied for even a $\mathrm{TeV}$ scale for left-right symmetry breaking. But the same Yukawa couplings enter in the expression for the generated $(B-L)$ asymmetry, which may then be very small. In this article we study systematically the Boltzmann equations for the generation of the lepton asymmetry and hence $(B-L)$ asymmetry to find out the lowest possible left-right symmetry breaking scale which satisfies the out-of-equilibrium condition and generates enough baryon asymmetry after the electroweak phase transition.

In the next section we briefly review the leptogenesis scenario, where one generates a lepton asymmetry when the right handed Majorana neutrinos decay. This then gets converted to a baryon asymmetry during the electroweak phase transition. Subsequently, we discuss the Boltzmann equations and the possible solutions for a low energy left-right symmetry breaking. 


\section{Model for leptogenesis}

It was first proposed by Fukugita and Yanagida [5] that in extensions of the standard model, which include singlet heavy right handed neutrinos, it is possible to generate a lepton asymmetry at some intermediate scale $\left(\sim 10^{10} \mathrm{GeV}\right)$, which can then get converted to a baryon asymmetry during the electroweak phase transition. The source of $\mathrm{CP}$ violation is in the mass matrix of the neutrinos. The out-of-equilibrium condition is satisfied with small Yukawa couplings. In this scenario the heavy right handed neutrinos decay to light left handed neutrinos in out-of-equilibrium. The amount of asymmetry thus generated depends on the amount of CP violation.

In this approach it is expected that at the time of the decay, there are enough right handed neutrinos given by their equilibrium distribution. However, in practice it is difficult to achieve this distribution [6]. Even though we assume that gravitational fields have generated equilibrium numbers of right handed neutrinos, during the period $10^{18} \mathrm{GeV} \rightarrow 10^{10} \mathrm{GeV}$ the equilibrium distribution cannot be maintained since the right handed neutrinos are sterile under the standard model and as the universe expands the number density reduces. When these fewer right handed neutrinos decay in out-ofequilibrium, they cannot generate enough lepton asymmetry.

It has been pointed out that if these right handed neutrinos take part in any gauge interaction, then the decays of the gauge particles can produce an equilibrium distribution of the right handed neutrinos. The left-right symmetric models are the natural extensions for this, where the heavy neutrinos interact with the right handed gauge bosons strongly [6]. As a result, the decay of the right handed gauge bosons can generate an equilibrium distribution of heavy neutrinos. In addition to the heavy neutrino decays, lepton number violating higgs decays also contribute to the generation of lepton asymmetry in these models [7].

In the following we shall thus consider a left-right symmetric extension of the standard model. We consider the symmetry breaking chain, $S U(3)_{c} \times$ $S U(2)_{L} \times S U(2)_{R} \times U(1)_{(B-L)}\left[\equiv G_{L R}\right] \stackrel{M_{R}}{\longrightarrow} S U(3)_{c} \times S U(2)_{L} \times U(1)_{Y}\left[\equiv G_{\text {std }}\right]$ 
$\stackrel{M_{W}}{\longrightarrow} S U(3)_{c} \times U(1)_{e m}$. The symmetry breaking $G_{L R} \rightarrow G_{s t d}$ takes place when the right handed triplet higgs field $\Delta_{R} \equiv(1,1,3,-2)$ acquires a vacuum expectation value $(v e v)$. In this model $(B-L)$ is a local symmetry. The breaking of the group $G_{L R}$ also implies spontaneous breaking of $(B-L)$. Left-right parity implies the existence of another higgs field $\Delta_{L}$ which transforms as $(1,3,1,-2)$ under $G_{L R}$. A higgs bi-doublet field $\phi(1,2,2,0)$ breaks the electroweak symmetry and gives masses to the fermions.

The fermion content of the model is, $q_{i L} \equiv[3,2,1,1 / 3], q_{i R} \equiv[3,1,2,1 / 3]$, $\ell_{i L} \equiv[1,2,1,1]$ and $\ell_{R} \equiv[1,1,2,1]$, where $i=1,2,3$ corresponds to three generations. The right handed neutrinos $\left(N_{i} \equiv \nu_{i R}\right)$ are contained in $\ell_{i R}$ and we do not have to include them by hand. The Yukawa couplings in the leptonic sector are given by,

$$
\mathcal{L}_{Y u k}=f_{i j} \overline{\ell_{i L}} \ell_{j R} \phi+f_{L i j} \overline{\ell_{i L}{ }^{c}} \ell_{j L} \Delta_{L}^{\dagger}+f_{R i j} \overline{\ell_{i R}}{ }^{c} \ell_{j R} \Delta_{R}^{\dagger} .
$$

The scalar potential has many more terms compared to the standard model. We write down only those terms which contribute to the generation of the lepton asymmetry of the universe,

$$
\mathcal{L}_{\text {int }}=g\left(\Delta_{L}^{\dagger} \Delta_{R} \phi \phi+\Delta_{L} \Delta_{R}^{\dagger} \phi \phi\right)+\text { h.c. }
$$

The vevs of these fields are not independent. We consider the minimum of the complete potential which satisfies $v_{L} \ll v_{R}$ and $v_{L} \approx v^{2} / v_{R}$, where $v_{L, R}$ and $v$ are the vevs of the fields $\Delta_{L, R}$ and $\phi$ respectively. We also assume that the left-right parity ( $D$ - parity) is not broken, and hence the masses of the fields $\Delta_{L}$ and $\Delta_{R}$ remains the same even after the breaking of $G_{L R}$, i.e., $m_{\Delta_{L}}=m_{\Delta_{R}}=m_{\Delta} \approx v_{R}$. At this scale $v_{R}$, the $(B-L)$ local symmetry is also broken by two units, which gives rise to Majorana masses of the neutrinos and neutron-antineutron oscillations.

The $\Delta_{L, R}$ can now decay into two leptons, while $\Delta_{L, R}^{\dagger}$ decay into two antileptons:

$$
\begin{aligned}
& \Delta_{L, R} \rightarrow \ell_{L, R}+\ell_{L, R} . \\
& \Delta_{L, R}^{\dagger} \rightarrow \ell_{L, R}^{c}+\ell_{L, R}^{c} .
\end{aligned}
$$


These interactions, along with the scalar interactions

$$
\begin{aligned}
\Delta_{L, R} & \rightarrow \phi+\phi, \\
& \rightarrow \phi^{\dagger}+\phi^{\dagger},
\end{aligned}
$$

give rise to lepton number violation. The interference of the tree level diagram and the one loop diagram of Fig. 1 can then give rise to a lepton asymmetry in the decay modes of (3) and (4) for the left-handed triplets $\Delta_{L}$ given by,

$$
\epsilon_{\Delta} \approx \frac{1}{8 \pi\left|f_{L i j}\right|^{2}} \operatorname{Im}\left[g^{*} f_{L i j}^{*} f_{i k} f_{j k}\right] F\left(\frac{g^{*}}{f_{R k k}}\right)
$$

where $F(q)=\ln \left(1+1 / q^{2}\right)$. The quantity $\left[g^{*} f_{L i j}^{*} f_{i k} f_{j k}\right]$ contains a $C P$ violating phase and so can be complex.

Although the left handed higgs triplets $\Delta_{L}$ can generate lepton asymmetry through their lepton number violating interactions, the scattering process $\Delta_{L}+\Delta_{L} \rightarrow W_{L}+W_{L}$ will make their number density the same as the equilibrium density below the GUT scale so, at low energies, they cannot satisfy the out-of-equilibrium condition. So for the generation of lepton asymmetry at low energy the main contribution comes from the heavy right handed neutrino decays. The vev of $\Delta_{R}$ spontaneously gives a Majorana mass to the right handed neutrinos. This in turn allows the decay of $\nu_{R}$ into a lepton and an antilepton,

$$
\begin{aligned}
N_{i} & \rightarrow \ell_{j L}+\bar{\phi}, \\
& \rightarrow \ell_{j L}^{c}+\phi .
\end{aligned}
$$

In the case of decays of the right-handed neutrinos there are two types of loop diagrams which can interfere with the tree level decays of (6) and (7) which are shown in Fig. 2. The interference of the tree level diagram and the one loop diagrams of Fig. 2 generates a lepton asymmetry given by,

$$
\epsilon_{\nu} \approx \frac{1}{4 \pi\left|f_{i k}\right|^{2}} \operatorname{Im}\left[f_{i k} f_{i l} f_{j k}^{*} f_{j l}^{*}\right] \frac{f_{R i i}}{f_{R k k}} .
$$

In addition to these one loop vertex type corrections, there are self energy type corrections [10, 11] which also contribute to the lepton asymmetry of 
the universe (Fig. 3). This contribution can also be viewed as a new indirect $C P$ violation entering in the mass matrix of the heavy neutrinos [11. This contribution becomes much stronger than the other contribution when the heavy neutrinos are almost degenerate.

When the mass squared difference between the two generations of heavy neutrinos are large, the interference of the tree level and the one loop diagrams of Fig. 3 has already been calculated in the literature [10]. This contribution has also been calculated in the other approach, where this is viewed as a new indirect $C P$-violation $\delta$, which enters through the mass matrix. Both the results come out to be the same and that confirms the equivalence between the two approaches. However, the latter approach has the advantage that it allows us to calculate the amount of $C P$ violation in the limit when the mass squared difference is very small.

In the case of a small mass difference, $\delta$ reads 11 :

$$
\delta=2 \pi g^{a b} \mathcal{C} \frac{M_{1} M_{2}}{M_{2}^{2}-M_{1}^{2}}
$$

where

$$
\left.\mathcal{C}=-\frac{1}{\pi} \operatorname{Im}\left[\sum_{\alpha}\left(f_{\alpha 1}^{*} f_{\alpha 2}\right) \sum_{\beta} f_{\beta 1}^{*} f_{\beta 2}\right)\right]\left(\frac{1}{\sum_{\alpha}\left|f_{\alpha 1}\right|^{2}}+\frac{1}{\sum_{\alpha}\left|f_{\alpha 2}\right|^{2}}\right)
$$

This contribution becomes significant when the two mass eigenvalues are close to each other. It indicates a resonance like behaviour of the asymmetry if the two mass eigenvalues are nearly degenerate. For very large values of the mass difference the two contributions $\epsilon_{\nu}$ and $\delta$ are of the same order of magnitude.

We now have to consider only the $\Delta_{L}, \Delta_{R}$ and $N_{1,2}$ decay processes. We assume $N_{1}$ to be the lightest of the right handed neutrinos. If the masses of $N_{1}$ and $N_{2}$ are almost degenerate, their decay widths can become larger than the mass difference. In this case both the neutrino decays will contribute to the lepton asymmetry of the universe. The decay widths for $\Delta_{L, R}$ and $N_{1}$ are,

$$
\Gamma_{\Delta_{L, R}}=\frac{\left|f_{[L, R] i j}\right|^{2}}{16 \pi} M_{\Delta} \quad \text { and } \quad \Gamma_{N_{i}}=\frac{\left|f_{1 j}\right|^{2}}{16 \pi} M_{N}
$$


where $M_{N}$ is the mass of $N_{1}$. Since we assumed $M_{N}<M_{\Delta}$, at low energy the $\Delta_{L}$ decay will erase all lepton asymmetry and then the $N_{1}$ decay will generate the required asymmetry. For this reason while working the details of the Boltzmann equation we take the effect of only $\epsilon=\epsilon_{\nu}$ and $\delta$. We shall now proceed to solve the Boltzmann equations including all these contributions and the scattering processes.

\section{Solutions of the Boltzmann equations}

The evolution of lepton and neutrino densities is governed by the Boltzmann equations. We start by deriving the Boltzmann equation for the neutrino number density $n_{i}$. The equation governing the evolution of $n_{i}$ is: [2]

$$
\begin{aligned}
\dot{n}_{i}+3 H n_{i}= & \int \mathrm{d} \Pi_{i} \mathrm{~d} \Pi_{1} \mathrm{~d} \Pi_{2}(2 \pi)^{4} \delta^{(4)}\left(p_{i}-p_{1}-p_{2}\right) \\
& \times\left\{-f_{i}\left(p_{i}\right)\left|\mathcal{M}_{0}\right|^{2}+\frac{1}{2}(1+\epsilon)\left|\mathcal{M}_{0}\right|^{2} f_{l}\left(p_{1}\right) f_{\Phi^{c}}\left(p_{2}\right)\right. \\
& \left.+\frac{1}{2}(1-\epsilon)\left|\mathcal{M}_{0}\right|^{2} f_{\bar{l}}\left(p_{1}\right) f_{\Phi}\left(p_{2}\right)\right\} \\
= & \int \mathrm{d} \Pi_{i} \mathrm{~d} \Pi_{1} \mathrm{~d} \Pi_{2}(2 \pi)^{4} \delta^{(4)}\left(p_{i}-p_{1}-p_{2}\right) \\
& \left\{-f_{i}\left(p_{i}\right)+f_{i}^{e q}\left(p_{i}\right)\right\}\left|\mathcal{M}_{0}\right|^{2}+\mathcal{O}(\epsilon, \mu / T) \\
= & -\Gamma_{i}\left(n_{i}-n_{i}^{e q}\right),
\end{aligned}
$$

where $n_{i}^{e q}$ is the equilibrium number density of the $N_{i}$, and $\Gamma_{i}$ is the thermally averaged decay width of $N_{i}$. The term on the left-hand side is the time derivative of $n_{i}$, plus a term which accounts for the dilution effect of the expansion of the universe. The integration is over phase space $\mathrm{d} \Pi$ and the phase space densities $f$, are given by the Maxwell-Boltzmann statistics:

$f_{i}(E)=\exp \left[-\frac{\left(E-\mu_{i}\right)}{T}\right], \quad f_{l}(E)=\exp \left[-\frac{(E-\mu)}{T}\right], \quad f_{\Phi}(E)=\exp \left[-\frac{(E+\mu)}{T}\right]$,

where $\mu$ is the chemical potential. The matrix element $\mathcal{M}_{0}$ is defined by:

$$
|\mathcal{M}(N \rightarrow \bar{l} \Phi)|^{2}=\left|\mathcal{M}\left(l \Phi^{c} \rightarrow N\right)\right|^{2}=\frac{1}{2}(1+\epsilon)\left|\mathcal{M}_{0}\right|^{2},
$$




$$
\left|\mathcal{M}\left(N \rightarrow l \Phi^{c}\right)\right|^{2}=|\mathcal{M}(\bar{l} \Phi \rightarrow N)|^{2}=\frac{1}{2}(1-\epsilon)\left|\mathcal{M}_{0}\right|^{2}
$$

where $\epsilon$ is a measure of $C P$-violation.

It is more convenient to work with the variables:

$$
Y_{i}=n_{i} / s, \quad x=M_{i} / T=[2 H(x=1) t]^{\frac{1}{2}},
$$

where $M_{i}$ is the mass of $N_{i} ; s=g_{*} n_{\gamma}$ is the entropy density of the universe; $g_{*}$ is the total spin degrees of freedom; $n_{\gamma}$ is the equilibrium photon density of the universe and $Y_{i}$ the number of neutrinos per co-moving volume element.

Thus:

$$
\frac{\mathrm{d} Y_{i}}{\mathrm{~d} x}=-K \gamma x\left(Y_{i}-Y_{i}^{e q}\right)
$$

where we have used:

$$
n_{\gamma}=s / g_{*}, \quad \frac{\mathrm{d} s}{\mathrm{~d} t}=-3 s \frac{\dot{R}}{R}=-3 s H,
$$

and:

$$
K=\frac{\Gamma_{i}(x=1)}{H(x=1)}, \quad \gamma=\frac{\Gamma_{i}(x)}{\Gamma_{i}(x=1)},
$$

with:

$$
Y_{i}^{e q}=n_{i}^{e q} / s=\left\{\begin{array}{ll}
g_{*}^{-1} & x \ll 1 \\
g_{*}^{-1} \sqrt{\pi / 2} x^{3 / 2} \exp (-x) & x \gg 1
\end{array} .\right.
$$

In solving the Boltzmann equations, we make the further change of variables, $X=g_{*} Y$, thus:

$$
\frac{\mathrm{d} X_{i}}{\mathrm{~d} x}=-K \gamma x\left(X_{i}-X_{i}^{e q}\right) .
$$

This is the Boltzmann equation for the evolution of neutrino number density, with the initial condition $X_{i}(0)=1$.

We now derive the Boltzmann equation for $L=\frac{1}{2}(l-\bar{l})$, where we have to take into account the processes $l+\Phi^{c} \leftrightarrow \bar{l}+\Phi$ mediated by a right-handed Majorana neutrino, as well as the processes:

$$
\bar{l} \leftrightarrow N \Phi^{c} .
$$


The Boltzmann equation for the number density of the light left-handed leptons is:

$$
\begin{aligned}
\dot{n}_{l}+3 H n_{l}= & \int \mathrm{d} \Pi_{N} \mathrm{~d} \Pi_{1} \mathrm{~d} \Pi_{2}(2 \pi)^{4} \delta^{(4)}\left(p_{N}-p_{1}-p_{2}\right) \\
& \times\left[-(1-\epsilon) f_{l}\left(p_{1}\right) f_{\Phi^{c}}\left(p_{2}\right)+(1+\epsilon) f_{N}\left(p_{N}\right)\right]\left|\mathcal{M}_{0}\right|^{2} \\
& +2 \int \mathrm{d} \Pi_{1} \mathrm{~d} \Pi_{2} \mathrm{~d} \Pi_{3} \mathrm{~d} \Pi_{4}(2 \pi)^{4} \delta^{(4)}\left(p_{1}+p_{2}-p_{3}-p_{4}\right) \\
& \times\left[-f_{l}\left(p_{1}\right) f_{\Phi^{c}}\left(p_{2}\right)\left|\mathcal{M}^{\prime}\left(l \Phi^{c} \rightarrow \bar{l} \Phi\right)\right|^{2}\right. \\
& \left.+f_{\bar{l}}\left(p_{3}\right) f_{\Phi}\left(p_{4}\right)\left|\mathcal{M}^{\prime}\left(\bar{l} \Phi \rightarrow l \Phi^{c}\right)\right|^{2}\right]+\mathcal{O}\left(\epsilon n_{l}+n_{l}^{2}\right) .
\end{aligned}
$$

The origin of the various terms is described below.

The first interaction term comes from direct $C P$-violation in the decay of the heavy Majorana neutrinos and is proportional to $\epsilon$, the $C P$-violating phase and the squares of the matrix element $\left|\mathcal{M}_{0}\right|$. The second term describes the generation of leptons from an initial neutrino state $\left|N_{i}\right\rangle$, with number density $n_{i}$. This converts into an antineutrino of a different generation $\left|N_{j}^{c}\right\rangle$ which then decays into a lepton and a higgs $\Phi$ with decay width:

$$
\Gamma_{j}=\frac{h_{\alpha j}^{2}}{16 \pi}
$$

The last term takes into account the $2 \leftrightarrow 2$ scattering mentioned above. The corresponding equation for $\bar{l}$ is obtained as usual, by interchanging $l \leftrightarrow \bar{l}$, $\epsilon \leftrightarrow-\epsilon$, etc. To obtain the Boltzmann equation for the evolution of $n_{L}=$ $\frac{1}{2}\left(n_{l}-n_{\bar{l}}\right)$ we subtract the equation for $n_{\bar{l}}$ from that for $n_{l}$ and multiply by a factor of $1 / 2$ :

$$
\dot{n}_{L}+3 H n_{L}=\epsilon \Gamma_{i}\left(n_{i}-n_{i}^{e q}\right)-n_{L}\left(n_{i}^{e q} / n_{\gamma}\right) \Gamma_{i}-2 n_{L} n_{\gamma}<\sigma|v|>,
$$

where $\left|\mathcal{M}^{\prime}\left(l \Phi^{c} \rightarrow \bar{l} \Phi\right)\right|^{2}$ and $\left|\mathcal{M}^{\prime}\left(\bar{l} \Phi \rightarrow l \Phi^{c}\right)\right|^{2}$ are the squares of the matrix elements for $2 \leftrightarrow 2 L$-nonconserving scatterings with the part due to real, intermediate-state $N$ 's removed. Here the quantity:

$$
\begin{array}{r}
<\sigma|v|>=\int \mathrm{d} \Pi_{1} \mathrm{~d} \Pi_{2} \mathrm{~d} \Pi_{3} \mathrm{~d} \Pi_{4}(2 \pi)^{4} \delta^{(4)}\left(p_{1}+p_{2}-p_{3}-p_{4}\right) \\
f_{l}\left(p_{1}\right) f_{l}\left(p_{2}\right)\left|\mathcal{M}^{\prime}\left(l \Phi^{c} \rightarrow \bar{l} \Phi\right)\right|^{2} / n_{\gamma}^{2},
\end{array}
$$


is the velocity-averaged $2 \leftrightarrow 2 L$-violating cross-section. The presence of the term $-\epsilon \Gamma_{i} n_{i}^{e q}$ is due to the $C P$-violating part of $\left|\mathcal{M}^{\prime}\left(l \Phi^{c} \rightarrow \bar{l} \Phi\right)\right|^{2}-\mid \mathcal{M}^{\prime}(\bar{l} \Phi \rightarrow$ $\left.l \Phi^{c}\right)\left.\right|^{2}$.

In parallel to the calculation for the $n_{i}$, we obtain:

$$
\frac{\mathrm{d} Y_{L}}{\mathrm{~d} x}=\epsilon K \gamma x\left(Y_{L}-Y^{e q}\right)-g_{*} Y^{e q} Y_{L} K \gamma x-\frac{2 Y_{L} \Gamma_{s} x}{H(x=1)}
$$

where $\Gamma_{s}=n_{\gamma}<\sigma|v|>$, with the initial condition $Y_{L}(0)=0$.

To solve the set of coupled differential equations, we take for $\Gamma_{i}, \gamma$ and $\Gamma_{s}$ :

$$
\begin{gathered}
\Gamma_{i}=\frac{f^{2}}{16 \pi} M_{i}\left\{\begin{array}{ll}
x & x \ll 1 \\
1 & x \gg 1
\end{array},\right. \\
\gamma=\left\{\begin{array}{ll}
x & x \ll 1 \\
1 & x \gg 1
\end{array},\right.
\end{gathered}
$$

and:

$$
\Gamma_{s}=\frac{3 f^{4}}{4 \pi} \frac{M_{i}}{x}\left\{\begin{array}{ll}
1 & x \ll 1 \\
\frac{2}{3} \frac{1}{x^{2}} & x \gg 1
\end{array} .\right.
$$

The functions $\gamma, \Gamma_{i}$, and $\Gamma_{s}$ must be specified in the region $x \approx 1$, in order to find a solution for the differential equations. We take:

$$
\begin{gathered}
\gamma=1-\exp (-x), \\
\Gamma_{i}=\frac{M_{i} f^{2}}{16 \pi}(1-\exp (-x)),
\end{gathered}
$$

and:

$$
\Gamma_{s}=\frac{3 f^{4} M_{i}}{4 \pi x}\left(1-\exp \left[-2 /\left(3 x^{2}\right)\right]\right),
$$

which are a good approximation to the functions (26), (27) and (28) above, in the regions $x \ll 1$ and $x \gg 1$. The numerical solutions to the Boltzmann equations are then obtained for different values of $f, K$ and $\epsilon$. We summarize our observations below.

For $K \ll 1$, the amount of lepton asymmetry grows cubically to a constant asymptotic value we call it $Y_{L}^{a s y m}(K \ll 1)$ as shown in figure 4 . In all these figures we have taken some representative values for the couplings to 
be of the order of 1 . The nature of the curve is independent of the choice of the Yukawa couplings. This asymptotic value is given by, $Y_{L}($ asym $)=\frac{\epsilon+\delta}{g_{*}}$. This is the case when the decay rate of $N_{1}$ is less than the expansion rate of the universe. In this case the scattering rates are also less than the expansion rate of the universe. If the mass difference between $N_{1}$ and $N_{2}$ is of the same order of magnitude to their masses, the contributions of $\epsilon$ and $\delta$ become comparable. This case has been discussed extensively in the literature and the constraint on the scale of $(B-L)$ breaking obtained from this condition is $M_{R}>10^{7} \mathrm{GeV}$ with the Yukawa couplings to be of the order of $10^{-5}$. Somewhat smaller values of the Yukawa couplings can reduce the scale of $(B-L)$ breaking to a lower value. However, then the amount of lepton asymmetry will also be inadequate, unless there is large hierarchy among the Yukawa couplings of different generations.

When the mass difference $\left|M_{1}-M_{2}\right| \sim 10^{-3} M_{1,2}, \delta$ can be three to four orders of magnitude larger than $\epsilon$. In this case we can consider $|f| \sim 10^{-7}$ and still get adequate amount of lepton asymmetry. This will then allow us somewhat smaller right handed symmetry breaking scale $M_{R}>10^{5} \mathrm{GeV}$.

When $K$ is of the order of unity or more, the lepton asymmetry vanishes before $T=M_{1}$. From $T=M_{1}$ onwards the lepton asymmetry starts increasing from its initial value of $Y_{L}=0$, but as it approaches the asymptotic value of $Y_{L}^{\text {asym }}(K \ll 1)$ the scattering processes becomes important and start depleting it exponentially (as shown in figures 5 and 6 ). Unlike the common folklore that when the system is in equilibrium the asymmetry falls exponentially fast, soon the scattering processes become unimportant and the lepton asymmetry reaches its new asymptotic value, which is less than $\frac{\epsilon+\delta}{g_{*}}$. For $K=1$ the suppression factor is about $1 / 5$ and for $K=5$ it is .02 . Figure 7 shows the fall of this asymptotic values of the lepton asymmetry for different values of $K$. For $K=1000$ the suppression factor is about $8 \times 10^{-6}$. In grand unified theories where the heavy gauge bosons decay generates an $(B+L)$ asymmetry it was shown [2] that this suppression factor is $\approx\left[K(\ln K)^{0.6}\right]^{-1}$. But in the case of leptogenesis, as figure 7 shows, this suppression is much faster than linear. For large mass difference $\left|M_{1}-M_{2}\right|, Y_{L}$ is approximately proportional to $f^{2}$ when all the $f^{\prime}$ 's are of the similar order of magnitude 
and $K \sim 10^{-3} f^{2} \frac{M_{P}}{M_{1}}$. Since $K$ and $Y_{L}$ both are proportional to $|f|^{2}$, we cannot improve the bound on the right handed symmetry breaking scale with large $K$. However, since the suppression for large $K$ is almost quadratic we can at most lower the scale of left-right symmetry breaking by one order of magnitude to about $10^{4} \mathrm{GeV}$.

For very large $K$, if $|f|^{4} \geq 10^{-17} M_{1}$, the scattering processes become larger than the expansion rate of the universe. In this case the lepton asymmetry decreases exponentially and never reaches any asymptotic value. In this region the actual equilibrium condition is satisfied.

\section{Summary and Conclusion}

We have studied the possible scale of left-right symmetry breaking, which can generate enough lepton asymmetry of the universe. For simplicity we assumed that the order of magnitude of the Yukawa couplings of the heavy neutrinos are similar. It was shown that the right handed symmetry breaking scale can be lowered to $10^{4} \mathrm{GeV}$ at most. Slight deviation from the out-ofequilibrium condition can still generate similar amount of leptons asymmetry, but that cannot change the scale of the symmetry breaking.

\section{Acknowledgment}

The work of S.L. is funded by a Marie Curie fellowship (TMR-ERBFMBICT950565) and that of J.F. and P.O'D by the Natural Sciences and Engineering Research Council of Canada. 


\section{References}

[1] A.D. Sakharov, Pis'ma Zh. Eksp. Teor. Fiz. 5 (1967) 32.

[2] E.W. Kolb and M.S. Turner, The Early Universe (Addison-Wesley, Reading, MA, 1989).

[3] M. Yoshimura, Phys. Rev. Lett. 41 (1978) 281; Erratum: Phys Rev. Lett. 42 (1979) 7461.

[4] V.A. Kuzmin, V.A Rubakov and M.E. Shaposhnikov, Phys. Lett. B 155 (1985) 36.

[5] M. Fukugita and T. Yanagida, Phys. Lett. B 174 (1986) 45.

[6] M. Plümacher, Z. Phys. C 74, 549 (1997).

[7] P.J. O’Donnell and U. Sarkar, Phys. Rev. D 49 (1994) 2118; W. Buchmuller and M. Plümacher, Phys. Lett. B 389, 73 (1996).

[8] P. Langacker, R.D. Peccei and T. Yanagida, Mod. Phys. Lett. A 1 (1986) 541; M.A. Luty, Phys. Rev. D 45 (1992) 455; A. Acker, H. Kikuchi, E. Ma and U. Sarkar, Phys. Rev. D 48 (1993) 5006.

[9] R.N. Mohapatra and X. Zhang, Phys. Rev. D 46 (1992) 5331; A. Ganguly, J.C. Parikh and U. Sarkar, Phys. Lett. B 385 (1996) 175.

[10] A. Yu. Ignatev, V.A. Kuzmin and M.E. Shaposhnikov, JETP Lett. 30 (1979) 688; F.J. Botella and J. Roldan, Phys. Rev. D 44 (1991) 966; J. Liu and G. Segre, Phys. Rev. D 48 (1993) 4609; L. Covi, E. Roulet and F. Vissani, Phys. Lett. B 384, 169 (1996).

[11] M. Flanz, E.A. Paschos and U. Sarkar, Phys. Lett. B 345 (1995) 248;

M. Flanz, E.A. Paschos, U. Sarkar and J. Weiss, Phys. Lett. B 389, 693 (1996). 

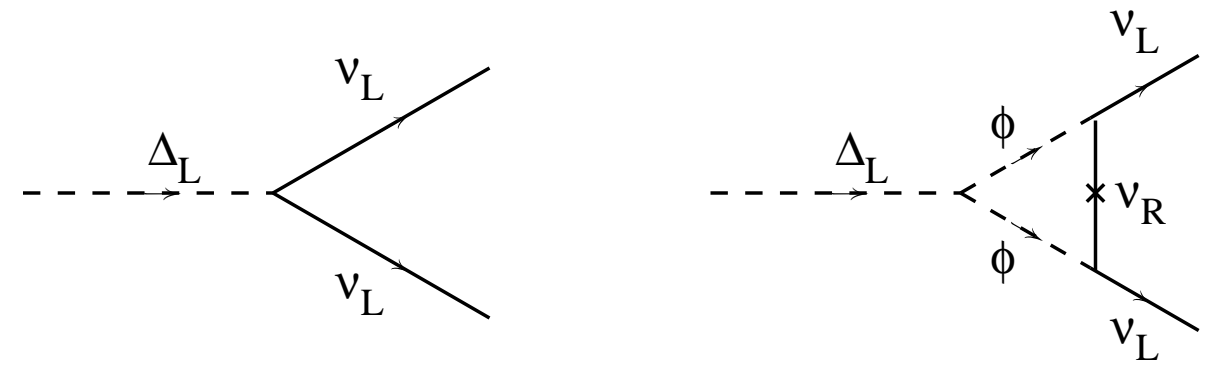

Figure 1: Tree and one loop diagrams of lepton number violating triplet higgs $\Delta_{L}$ decay 

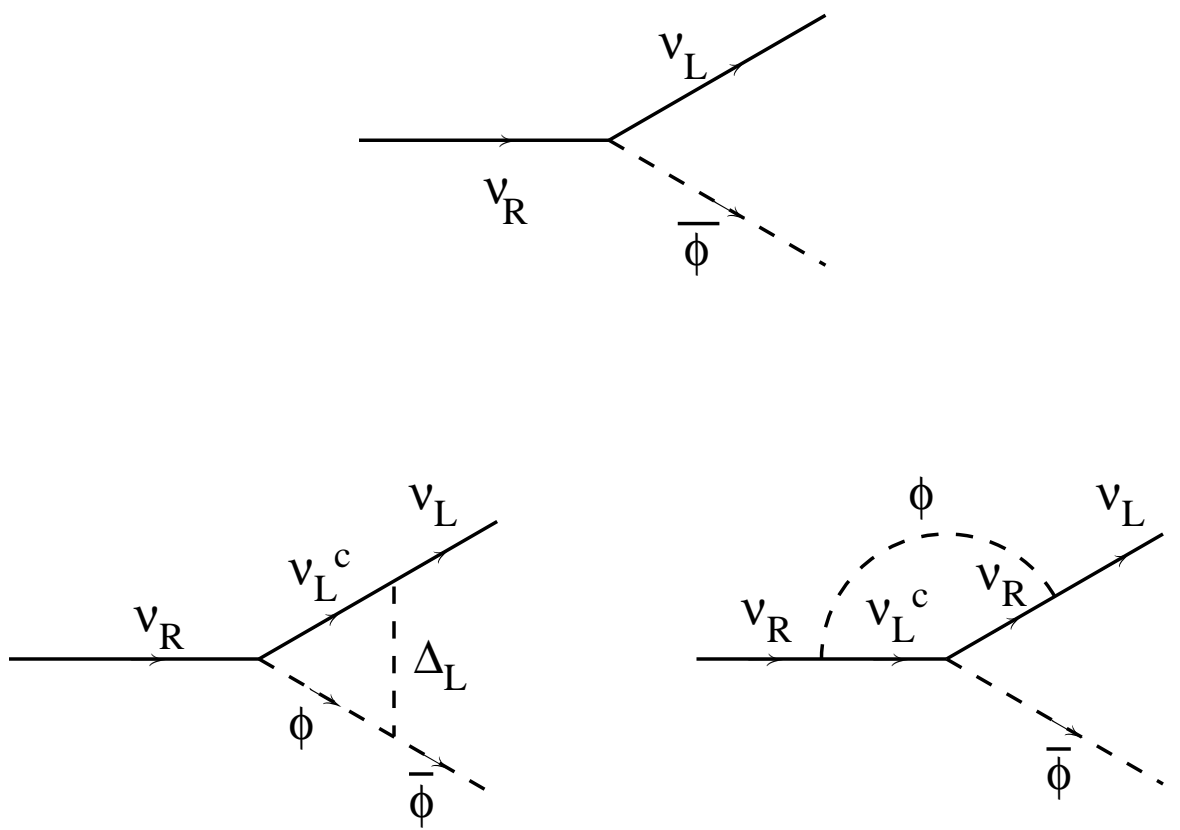

Figure 2: Tree and vertex correction type one loop diagrams contributing to the generation of lepton asymmetry 

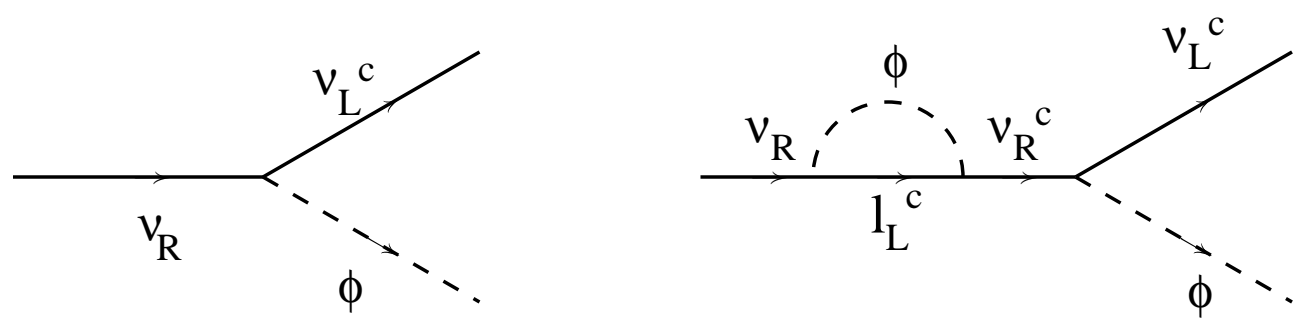

Figure 3: Tree and self energy correction type one loop diagrams contributing to the generation of lepton asymmetry 


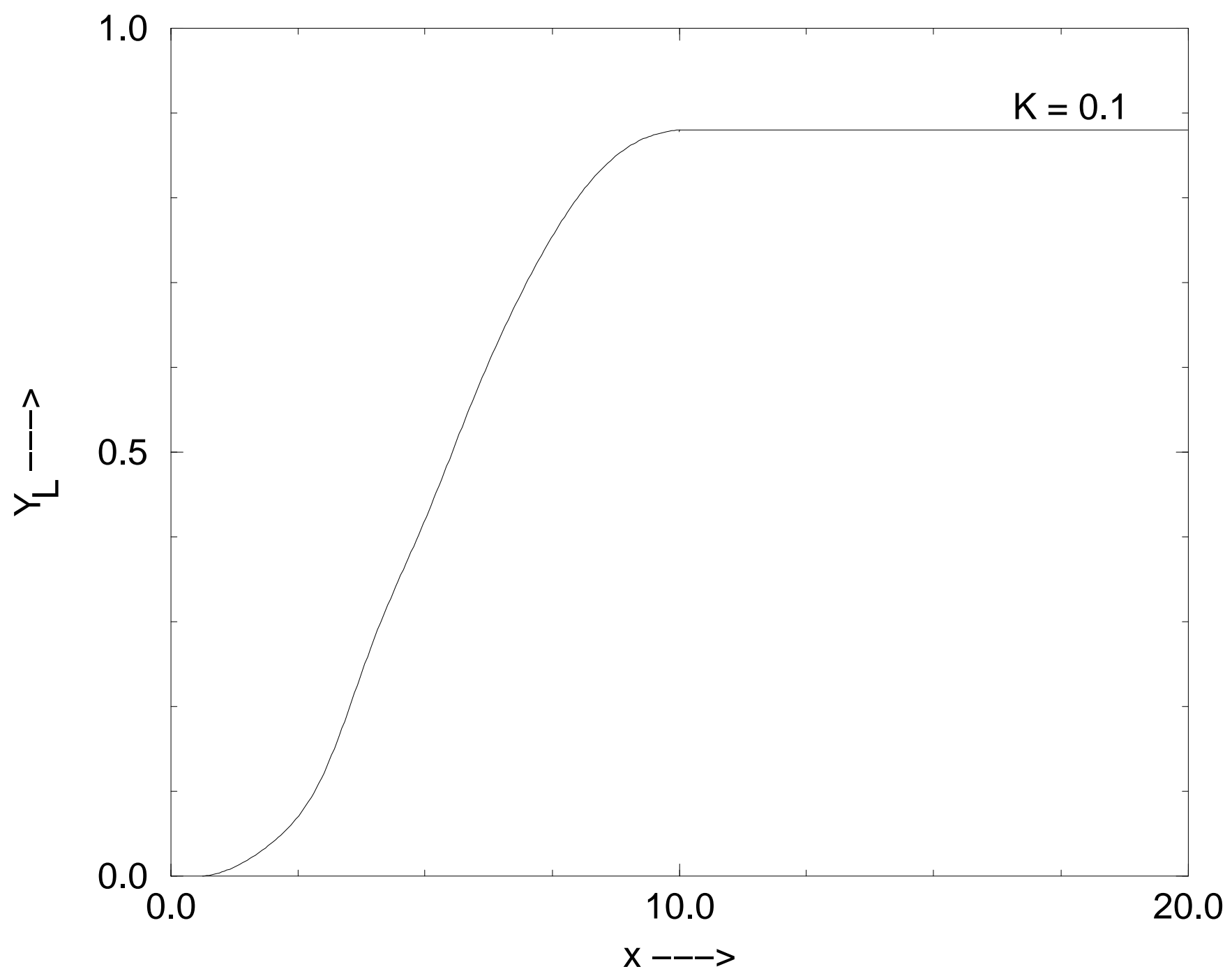

Figure 4: Lepton asymmetry $Y_{L}$ grows steadily to a constant asymptotic value $\epsilon$ for $K<1$. 


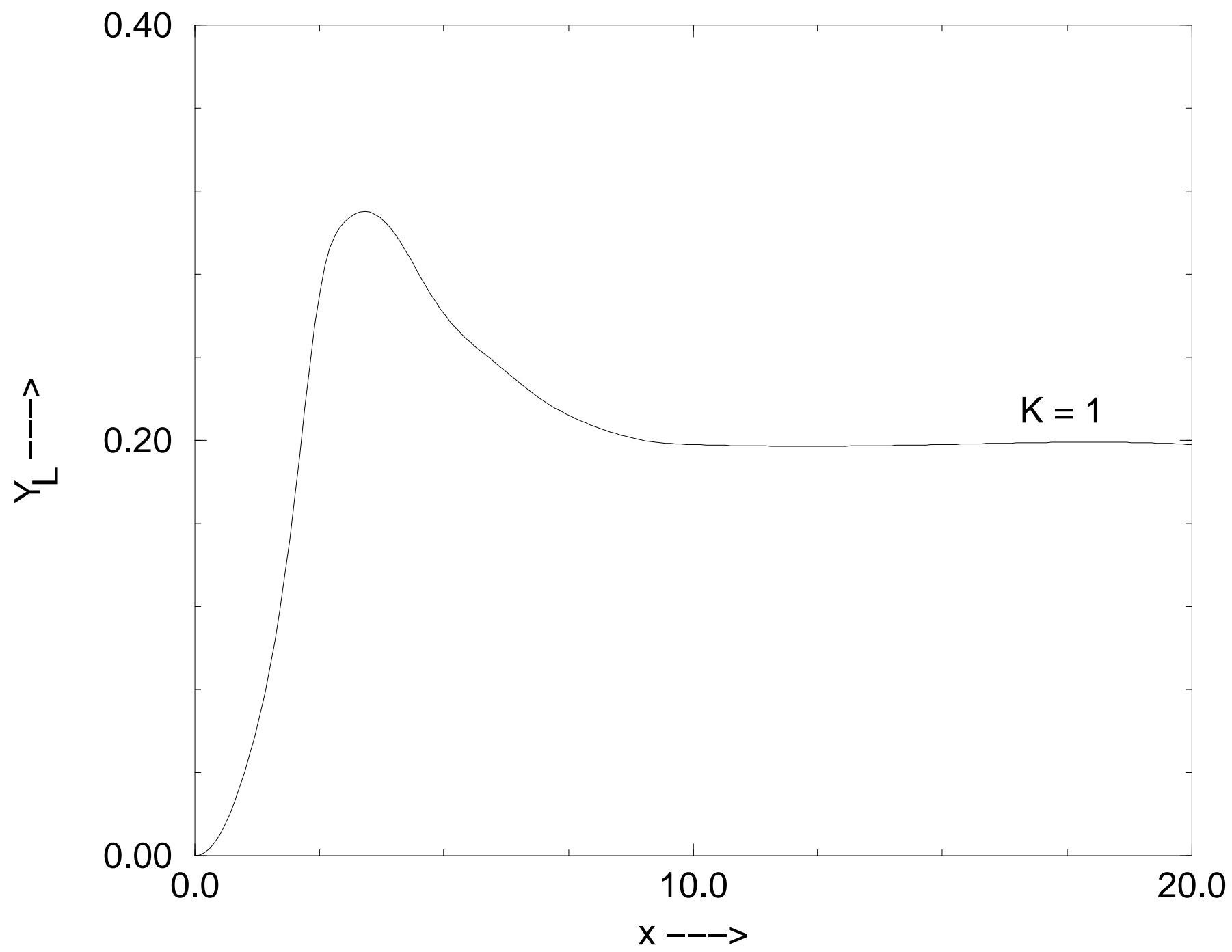

Figure 5: For $K=1$, lepton asymmetry starts depleting before reaching a constant value. The asymptotic constant value is thus much less than $\epsilon$. 


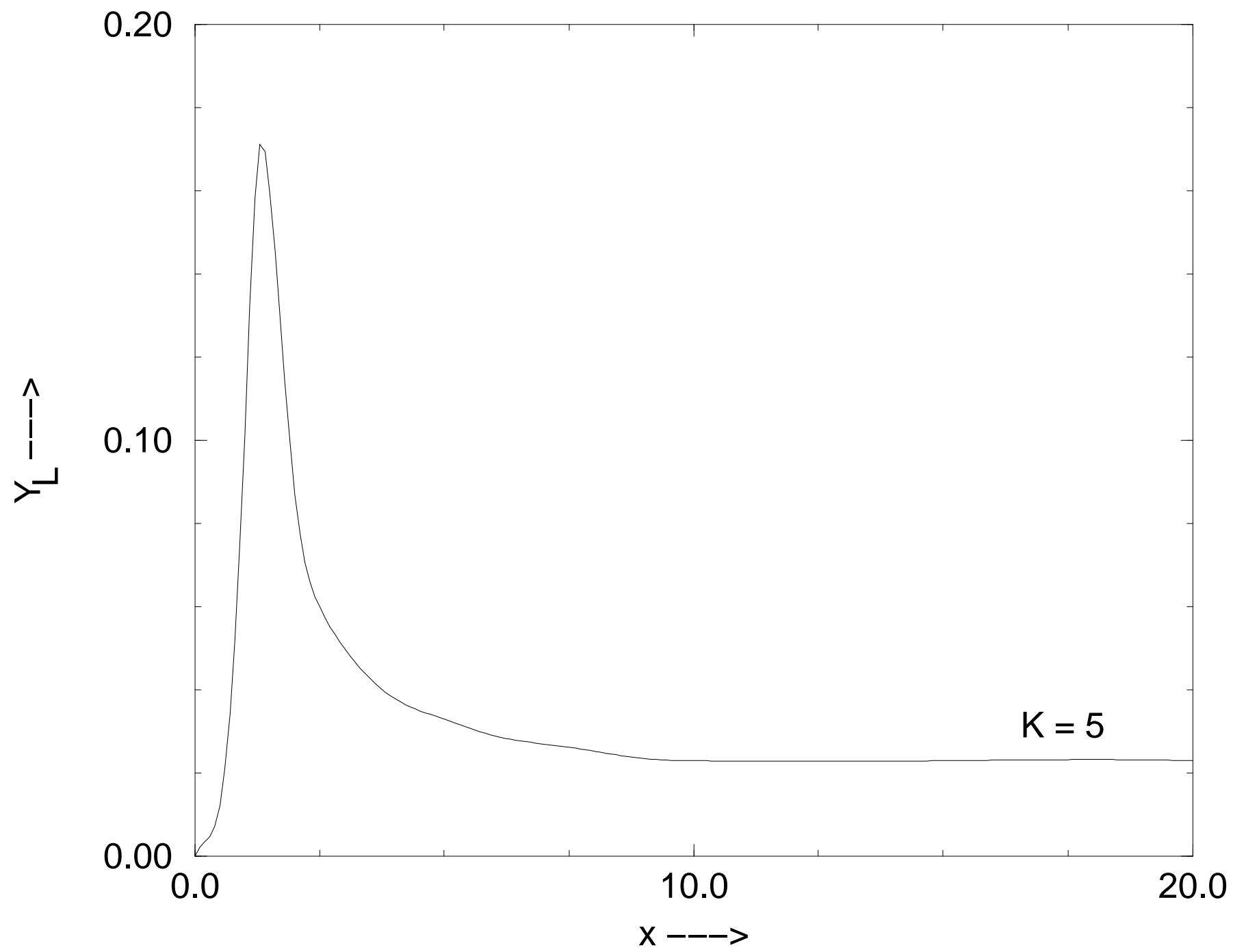

Figure 6: For large $K>1$ the behaviour is similar to $K=1$. For $K=5$ the asymptotic value is further depleted. 


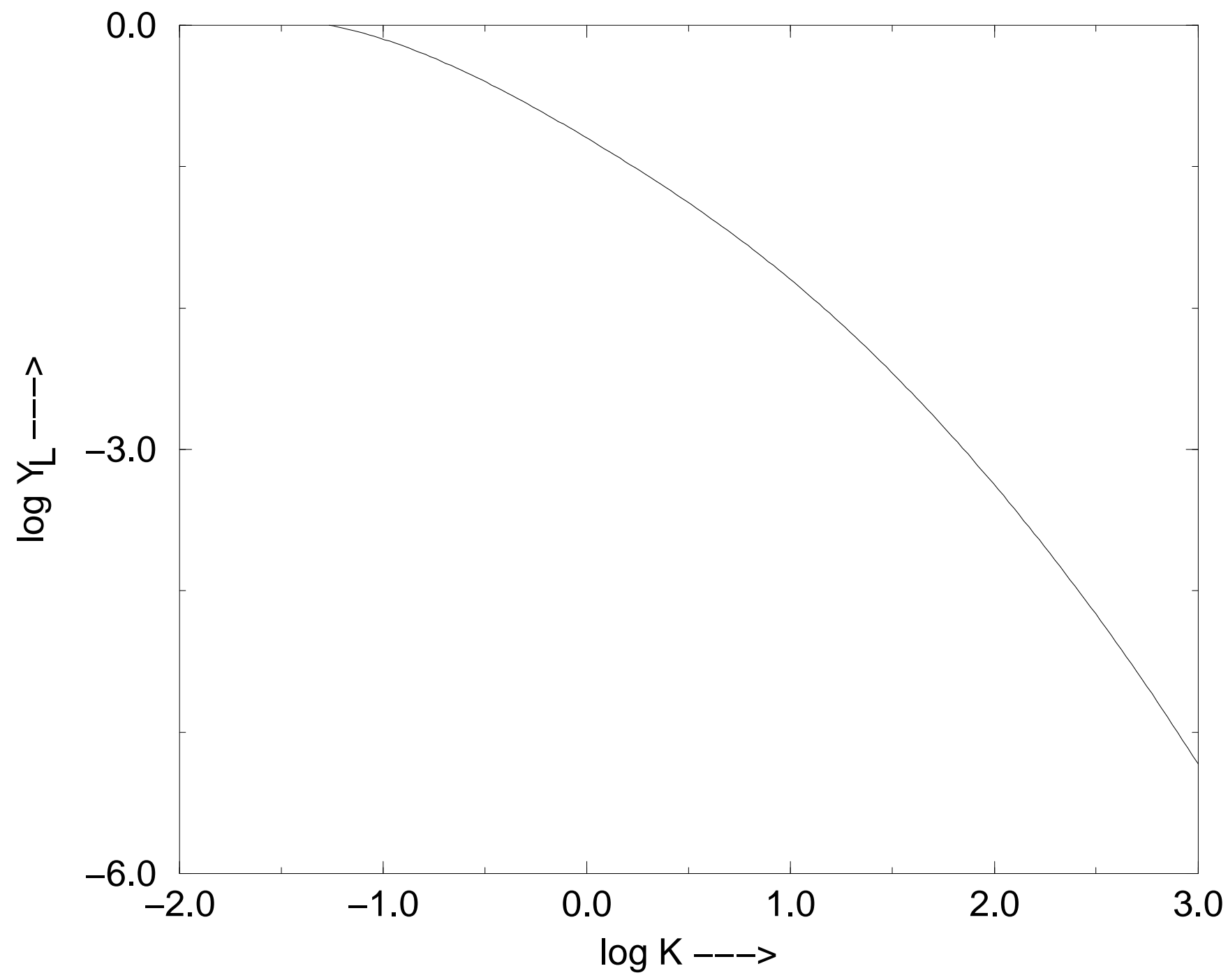

Figure 7: The asymptotic value of the lepton asymmetry for different values of $K$ in $\log _{10}-\log _{10}$ graph. For $K=1000$ the lepton asymmetry drops to $8 \times 10^{-6}$. 